\title{
Naturaleza privada y calidad de vida. Influencia de la naturaleza doméstica en el bienestar de los propietarios de las casas con patio de la ciudad de Córdoba, España ${ }^{1}$
}

\author{
Carlos Priego González de Canales², Jürgen Breuste ${ }^{3}$ y Luis Rodríguez- \\ Morcillo Baena ${ }^{4}$
}

\begin{abstract}
RESUMEN
Numerosas investigaciones consideran que los espacios verdes públicos de las ciudades son lugares privilegiados para que se desarrollen los procesos de socialización e interacción entre los ciudadanos y la naturaleza. Sin embargo, este trabajo demuestra que los ciudadanos que viven en determinados espacios privados, como las casas con patio del barrio histórico de Córdoba, pueden llegar a percibir tantos o más beneficios sociales en sus patios, que si visitaran otros espacios verdes de la ciudad. Para desarrollar este trabajo se utilizó una metodología cualitativa; utilizando la técnica de los grupos de discusión y una metodología cuantitativa; mediante la realización de una encuesta presencial. La investigación muestra que los patios de la ciudad de Córdoba son los espacios verdes mayormente escogidos por sus propietarios para pasar su tiempo libre, y que el contacto y cuidado de estos pequeños espacios contribuyen a generar en sus propietarios y usuarios una satisfacción y nivel de bienestar igual o incluso superior al provocado si visitaran otros espacios verdes de la ciudad.
\end{abstract}

Palabras clave: Naturaleza privada, calidad de vida, patios, naturaleza urbana, espacios verdes urbanos.

\begin{abstract}
Numerous studies consider public green spaces in cities to be privileged places for the socialization and interaction processes that are developed between people and nature. However, our work shows that people living in certain private areas, such as houses with courtyards in the historic quarter of Cordoba, may receive as many or more benefits in their courtyards, than if they visited other green spaces in the city. To develop this concept we used a qualitative methodology, focus groups, as well as quantitative methodology, face to face interviews. Research shows that private courtyards in the city of Cordoba are most commonly chosen green space for the owners to spend their free time, and that contact and care of these small spaces, all contribute to the owners and users level of satisfaction. This satisfaction is equal to or greater than the effect if they visited other urban green spaces.
\end{abstract}

Key words: private nature; quality of life; courtyards; urban nature; urban green spaces.

\footnotetext{
1 Artículo recibido el 20 de marzo de 2012, aceptado el 27 de agosto de 2013 y corregido el 10 de septiembre de 2013.

2 Departamento de Didáctica de las Ciencias Sociales y Experimentales. Grupo de Investigación: Didáctica de las Ciencias Experimentales. Universidad de Córdoba (España). E-mail: carlospgdc@hotmail.com
}

\footnotetext{
3 Departamento de Geografía, Geología y Mineralogía, Universidad Paris-Lodron de Salzburgo (Austria). E-mail: juergen.breuste@sbg.ac.at

4 Instituto de Estudios Sociales Avanzados (IESA), Consejo Superior de Investigaciones Científicas (España). E-mail: Irodriguez@iesa.csic.es
} 
En las últimas décadas se han realizado numerosos esfuerzos para proteger la naturaleza, sobre todo aquella naturaleza que, debido a su riqueza, biodiversidad, estado de conservación y soporte al ecosistema local o regional, aporta importantes beneficios a la sociedad. En estos casos, los beneficios "físicos" de los espacios naturales (extensión, tipo, número de especies, calidad de conservación, etcétera) predominan sobre los beneficios "sociales" (relax, conciencia ambiental, satisfacción, etcétera) a la hora de considerar su inclusión en las listas de protección.

La existencia de espacios verdes en las ciudades es un factor de suma importancia en la sostenibilidad de la ciudad, entendida como una combinación de las condiciones físicas del medio ambiente (calidad del aire y del agua, nivel de ruido, grado de contaminación, etcétera) y de los niveles de bienestar alcanzados en la persona (salud, relax, autoestima, socialización, etcétera) (Pacione, 2003; Hills, 1995; Benzeval et al., 1995). Por otro lado, habría que aclarar que aunque el actual concepto de "sostenibilidad" está cada vez está más asociado a un modelo de ciudad, que integra variables económicas, sociales y ambientales, no siempre profundiza en aspectos tan importantes como las experiencias, la percepción, satisfacción, e identidad de los ciudadanos, entre otras (Beer, 1994; Chiesura, 2004).

La importancia de la naturaleza urbana en las ciudades, radica en los efectos positivos y en las numerosas interacciones que tienen sobre la población residente (Matsuoka \& Kaplan, 2008). Estos efectos ayudan a incrementar la calidad de vida y pueden manifestarse desde diferentes ámbitos. Desde la "Componente Ambiental", los espacios verdes urbanos pueden afectar directa o indirectamente a la calidad del aire a nivel local o regional (Camilloni \& Barros, 1997), intervienen en la disminución de los contaminantes atmosféricos (Smith, 1978; 1990), en la conservación del agua y la reducción de la erosión del suelo (Peck \& Callaghan, 1999), en la disminución de la contaminación acústica (Miller, 1988) (Cook, 1978; Cook \& Haverbeke, 1971) y el aumento de la biodiversidad, entre otros. Respecto a la "Componente So- cial o Humana", los espacios verdes urbanos ejercen numerosos efectos positivos sobre la población residente, efectos que, según Matsuoka y Kaplan (2008), pueden clasificarse en dos grandes grupos. Un primer grupo estaría relacionado con las características físicas de la naturaleza urbana, y puede categorizarse en términos de contacto con la naturaleza, preferencias estéticas y la recreación y el juego. Los diferentes tipos de equipamientos naturales y el estado de conservación de las áreas residenciales contribuyen de forma importante a la apreciación que los vecinos hacen de sus barrios (Crow, et al., 2006). Así como la belleza escénica, la limpieza, los equipamientos, o la presencia de agua, en forma de lagunas o ríos, pueden ser vistos como categorías de preferencias estéticas. Todas estas preferencias por los espacios verdes pueden estar representadas en los jardines y parques (Jim \& Chen, 2006; Özgüner \& Kendle, 2006), en los corredores verdes (Gobster, 1995), o en los barrios con zonas verdes (Kaplan \& Austin, 2004; Crow et al., 2006; Ellis et al., 2006).

Un segundo grupo dentro de la Componente Social, incluiría la participación ciudadana en el proceso de diseño, la identidad de la comunidad con su entorno y la creación de la conciencia ambiental. La mera presencia de zonas verdes en las ciudades, representa una importante oportunidad para que la población aprenda sobre los principios ecológicos y sus interconexiones, ya que permite un aprendizaje práctico sobre el funcionamiento de los ecosistemas (Hough, 1989). Miles et al., (1998) señalaron que trabajar en contacto directo con la naturaleza constituye, a iguales condiciones laborales, una gran fuente de satisfacción para los trabajadores. Puede decirse, por tanto, que la presencia de árboles y plantas es un poderoso elemento de identificación de los residentes de un barrio con sus espacios públicos, favoreciendo la construcción de valores comunes y creando vínculos sociales entre los ciudadanos (Lewis, 1992). Esta idea conecta directamente con los enfoques del capital social aplicados a las áreas urbanas. La contribución de la naturaleza a la creación de capital social, viene avalada por los estudios de Lewis (1996), Berman (1997) y Kuo et al. (1998), entre otros, que demostra- 
ron cómo el vecindario residente en barrios con programas de urbanización basados en la creación de áreas verdes aumenta sus interacciones sociales y desarrolla un alto sentido de identidad y valores compartidos, al tiempo que experimenta un elevado nivel de gratificación.

Respecto a las relaciones de los ciudadanos con su medio natural más cercano, los estudios de Breuste et al. (2003) y de Priego et al. (2009) analizan la satisfacción que tienen los ciudadanos con los espacios verdes públicos que les rodean. En dichos estudios, los autores concluyen que tanto la cultura como el estrato socioeconómico del usuario son variables que influyen en el uso y el disfrute que los ciudadanos hacen de dichos espacios, por lo que tendrían que ser considerados en la planificación de los espacios verdes urbanos.

En este contexto, cada vez adquiere más importancia, en los debates públicos, la idea de que el desarrollo sostenible de las ciudades tiene que estar directamente relacionado con el grado de satisfacción subjetiva que tienen los residentes con su propio entorno. Apoyados en esta idea, algunas investigaciones empezaron a sugerir estándares mínimos de superficies de espacios verdes urbanos que deberían tener las ciudades, no obstante, la Organización Mundial de la Salud (OMS) considera entre 9 y 15 metros cuadrados la cantidad necesaria para cada habitante.

Guiados por estos indicadores internacionales, las ciudades empezaron a replantear su planificación urbana. Muchas de ellas con el afán de encabezar la lista de ciudades con el mayor número de metros cuadrados de espacios verdes por habitante, y por consiguiente, autoproclamarse ciudades sostenibles. Sin embargo, y al margen de los debates en el análisis de la metodología utilizada para cuantificar los espacios verdes urbanos, podríamos decir que, en la actualidad, no existe una clasificación consensuada que indiqué qué espacios podrían incluirse en el listado de espacios verdes a analizar.

Muchos estudios vienen a demostrar los numerosos beneficios que los espacios ver- des urbanos aportan al ciudadano, hagan o no hagan los ciudadanos uso de estos. Sin embargo, y en el contexto de esta investigación, podemos concluir que son pocos los estudios que analizan y relacionan el uso que la población hace de la naturaleza privada en sus casas y la satisfacción que esta les reporta.

Para llevar a cabo la investigación se planteó como objetivo general demostrar cómo, en determinados casos, el contacto y uso de los espacios verdes privados puede llegar a ser igual, o incluso más satisfactorio que el uso de otros espacios verdes públicos. Demostrando cómo, para los propietarios de las casas con patio, el uso de estos espacios verdes privados puede ser prioritario frente al uso de los espacios verdes públicos.

El trabajo se basa en una investigación que intenta demostrar cómo los ciudadanos que viven en casas con patio del barrio histórico de Córdoba, además de estar en continuo contacto con la naturaleza, pueden llegar a desarrollar intensas relaciones sociales vecinales, y por lo tanto, una identidad y satisfacción personal más fuertes que si vivieran en otro tipo de vivienda de la ciudad, o si visitaran otro tipo de espacios verdes de la ciudad.

Junto a este objetivo general se fijaron también los siguientes objetivos específicos:

- Conocer las diferencias existentes entre la población que vive en casas con patios y los que no viven en este tipo de viviendas, respecto al significado e importancia de la naturaleza en sus casas.

- Conocer las actitudes generales de la población hacia los patios, en sus aspectos convivenciales, sociales, ecológicos y festivos.

- Conocer las preferencias que tienen los ciudadanos para pasar su tiempo libre en los diferentes espacios verdes urbanos que tiene la ciudad.

De esta forma, esta investigación demuestra cómo en determinados lugares, culturas y tradiciones, el uso de la naturaleza privada puede aportar iguales o incluso mayores beneficios sociales en sus usuarios que el uso de los espacios públicos. Esta investigación 
viene a demostrar, por tanto, cómo los propietarios de las casas con patio de la ciudad de Córdoba prefieren pasar su tiempo libre en estos espacios verdes privados frente a los espacios públicos de la ciudad.

\section{Descripción de los patios de la ciudad de Córdoba y metodología de estudio}

En el caso de la ciudad de Córdoba (España), el casco histórico agrupa algo más de 14.000 viviendas de las que en su mayor parte son viviendas pequeñas, entre los 150$200 \mathrm{~m}^{2}$, con la característica agregación de los tejidos de raíz islámica, predominando las tradicionales casas con patios. Una parte significativa de estas viviendas son unifamiliares (algo más de 2.200), siendo las restantes plurifamiliares, con una media de cinco viviendas por edificio (Ayuntamiento de Córdoba, 2009)

Podemos afirmar que estas casas con patios son manifestaciones de sociabilidad, es decir, traspasan las barreras arquitectónicas para convertirse en expresiones culturales, constituyendo de manera articulada un modelo de vida propio de un entorno urbano específico, que se encuentra entre lo privado y lo público.

Aunque los patios tienen orígenes griegos han estado fuertemente influenciados por la cultura romana, especialmente en el sur de España, siendo Córdoba, por su importancia romana y por su ubicación geográfica, un lugar donde se desarrollan los patios en todos los órdenes, desde patios en las casas palaciegas hasta en las casas humildes.

Con la llegada de los árabes a Córdoba, el patio cobró una mayor importancia, incorporando nuevos elementos, como las fuentes de agua y la vegetación, que hacían coincidir este espacio de la casa con la imagen que tenían del paraíso musulmán. Es desde entonces cuando el patio empieza a tener una función social, transformándose en un lugar socializador, donde el patio representa la libertad de poder hablar con los familiares y vecinos al aire libre, y donde se disfruta de la naturaleza sin necesidad de salir a la calle.

Figura $\mathrm{N}^{\circ} 1$

Tipología edificatoria de los patios de Córdoba.

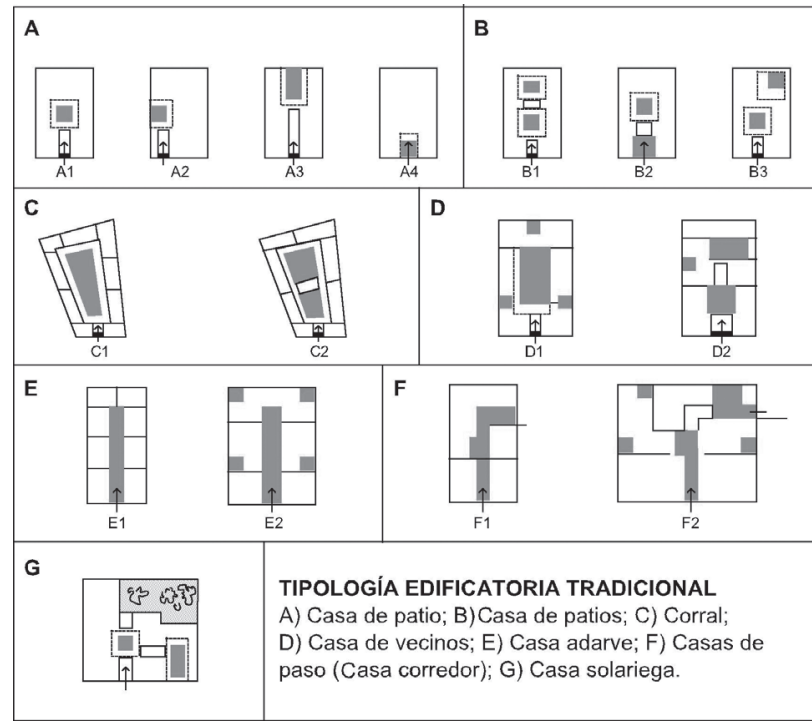

Fuente: Ayuntamiento de Córdoba. 
Hasta la fecha, el patio cordobés no ha evolucionado mucho, pero se distingue por su variedad: una primera modalidad estaría compuesta por aquellos patios que se encuentran en los palacios señoriales, conventos o antiguos hospitales; una segunda modalidad referida a las casas con patios de nueva construcción y una tercera a los patios de las casas más populares (Figura $N^{\circ} 1$ ).

Además de que las casas con patios de Córdoba (Figuras Nos 2-5), representan una forma de vida única en el mundo, son la base de una de las fiestas más populares de la primavera de esta ciudad. La fiesta de

Figura $\mathrm{N}^{\circ} 2$

Casa Unifamiliar

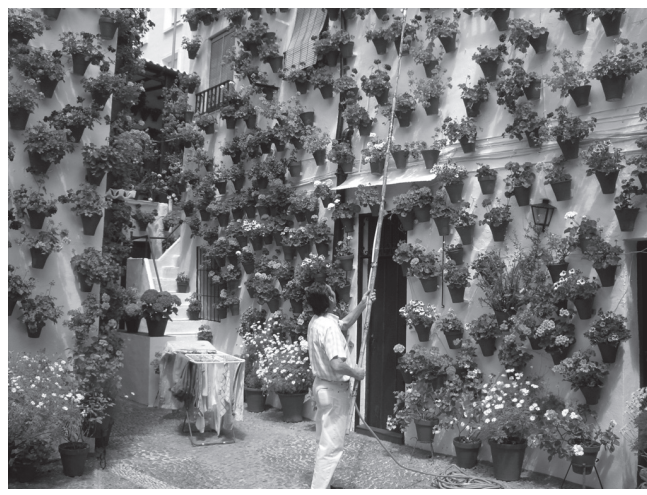

Fuente: Archivo propio del autor.

Figura $\mathrm{N}^{\circ} 4$

Casa de vecinos

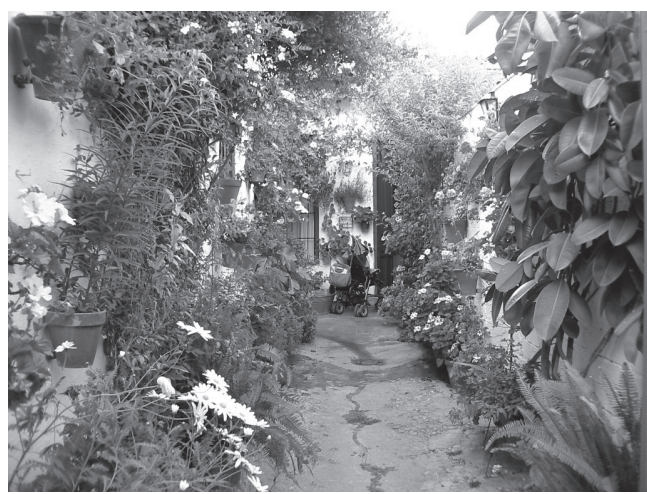

Fuente: Archivo propio del autor. los patios de Córdoba, que nació en 1918, surgió de una forma espontánea como consecuencia de la llegada a la ciudad de una población rural, que instauró una forma de vida sencilla y comunitaria. La fiesta de los patios de Córdoba es el máximo exponente de la vida social de sus habitantes, ya que durante unas semanas del mes de mayo, los habitantes de las casas con patios abren sus hogares a los visitantes, para que puedan observar el esplendor de sus plantas, así como un modelo de vida que se transmite a lo largo de los siglos y que se desarrolla en pleno casco histórico cordobés.

Figura $\mathrm{N}^{\circ} 3$

Casa Unifamiliar

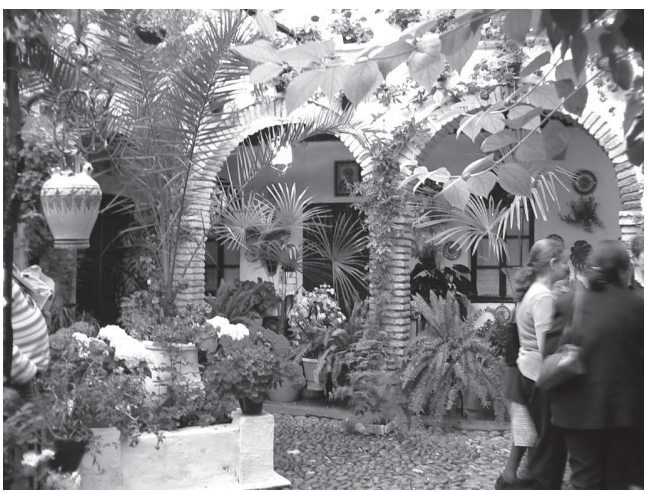

Fuente: Archivo propio del autor.

Figura $\mathrm{N}^{\circ} 5$

Casa de vecinos

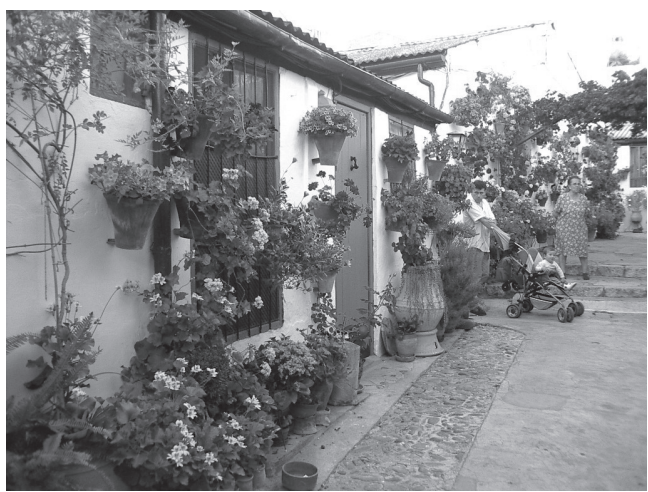

Fuente: Archivo propio del autor. 
Por todo esto, el 6 de diciembre de 2012, la fiesta de los patios de Córdoba fue declarada por la UNESCO Patrimonio Inmaterial de la Humanidad. En este contexto, y siguiendo las investigaciones que estudian la relación del ciudadano con su entorno más inmediato, el estudio de la percepción y valoración de la naturaleza privada de los patios de Córdoba adquiere un mayor interés como medio para descubrir, analizar y explicar la articulacióndesarticulación, y la integración-desintegración de una población local compleja, afectada por procesos de transformación urbanísticos, demográficos y socioeconómicos propios de todos los cascos históricos. En el caso de los patios de la ciudad de Córdoba, el contacto con la naturaleza privada adquiere un especial protagonismo, ya que influye de manera considerable en el desarrollo de las relaciones sociales y personales, en el aumento de la satisfacción del individuo y, como consecuencia, en el aumento de la calidad de vida de sus usuarios. Todos estos aspectos hacen que los patios de Córdoba constituyan un elemento cultural diferenciador respecto al resto de España y del mundo.

Para satisfacer los objetivos anteriormente expuestos, y debido a la complejidad de la investigación a desarrollar, así como el escaso conocimiento previo sobre este tema, se consideró apropiado utilizar una metodología dual de investigación: i) Metodología de tipo cualitativa; utilizando la técnica de los grupos de discusión, y ii) Metodología de tipo cuantitativo; mediante la realización de una encuesta presencial a una muestra de 20 propietarios de casas con patio de la ciudad de Córdoba.

En función de los objetivos de la investigación se realizaron 5 grupos de discusión (Cuadro $N^{\circ} 1$ ) en el mes de octubre de 2008. Las variables estructurales más importantes que se tuvieron en cuenta para el diseño de los grupos fueron tres: i) vivir en una casa con patio, y por consiguiente residir en el casco histórico de Córdoba, o por el contrario, no tener una casa con patio y vivir fuera del casco histórico; ii) presentación o no presentación de la casa con patio al concurso de "Patios Cordobeses"; y iii) la edad y el sexo de los participantes.
No habitantes en casas con patios. No residentes en el casco histórico

Entre los no residentes en el casco histórico se escogieron dos subgrupos diferenciados por la edad de sus participantes. Un primer subgrupo de jóvenes con edades comprendidas entre los 22 y 30 años (Grupo Discusión 1) y otro de adultos mayores entre 45 y 60 años (Grupo de Discusión 2).

\section{Habitantes en casas con patios.} Residentes en el casco histórico

Entre los residentes en el casco histórico se diferenciaron los ciudadanos que se presentan al concurso de patios y los que no lo hacen. Esta segunda variable dicotómica se consideró relevante ya que la participación en el concurso supone un plus de implicación y dedicación con la ornamentación de los patios, y porque además viven de modo más directo los costes y beneficios asociados con esa participación.

Entre los ciudadanos que residen en el casco histórico y que presentan sus patios al concurso anual, se tuvo en cuenta la variable edad, formándose un grupo con participantes de entre 35 y 50 años (Grupo Discusión 3), y otro grupo con mayores de 50 años (Grupo Discusión 4). Por último, se constituyó un grupo de discusión entre ciudadanos residentes en el casco histórico, con edades comprendidas entre 40 y 55 años y que nunca hubieran presentado su patio al concurso (Grupo Discusión 5).

Con esta investigación, aspectos como el uso de la naturaleza en los hogares, el significado que tiene para los propietarios, la percepción y satisfacción que les reporta, y la influencia que tiene en las relaciones sociales, tanto familiares como vecinales, son estudiados con mayor profundidad.

\section{El patio como lugar de contacto con la naturaleza y aumento de la calidad de vida}

El significado del patio como un entorno natural es general en todos los grupos y personas entrevistadas. Tener un patio es estar 


\section{Cuadro $\mathrm{N}^{\circ} 1$}

Características de los grupos de discusión.

\begin{tabular}{|c|c|c|}
\hline \multicolumn{3}{|c|}{ No residentes en casas con patio ni en el casco histórico } \\
\hline Grupo 1 & $\begin{array}{l}\text { Edad: Entre } 22 \text { y } 30 \text { años } \\
\text { Tamaño grupo: } 5 \text { individuos } \\
\text { Sexo: } 3 \text { hombres, } 2 \text { mujeres } \\
\text { Residencia: Fuera del Casco histórico }\end{array}$ & $\begin{array}{l}\text { Nivel de estudios: Mínimo bachiller o FP } \\
\text { II o Ciclo formativo de FP. Máximo Diplo- } \\
\text { mado Universitario o FPIII o ciclo de FP } \\
\text { Superior } \\
\text { Ocupación: Activos (Parados u ocupados). }\end{array}$ \\
\hline Grupo 2 & $\begin{array}{l}\text { Edad: Entre } 45 \text { y } 60 \text { años } \\
\text { Tamaño grupo: } 6 \text { individuos } \\
\text { Sexo: } 2 \text { hombres, } 4 \text { mujeres } \\
\text { Residencia: Fuera del casco histórico }\end{array}$ & $\begin{array}{l}\text { Nivel de estudios: Mínimo EGB o Estudios } \\
\text { Primarios. Máximo bachiller o FP II o Ciclo } \\
\text { formativo de FP } \\
\text { Ocupación: Activos (Parados u ocupados) o } \\
\text { Pasivos (amas de casa o pensionistas). }\end{array}$ \\
\hline \multicolumn{3}{|c|}{ Residentes en casas con patio y en el casco histórico } \\
\hline Grupo 3 & $\begin{array}{l}\text { Edad: Entre } 35 \text { y } 50 \text { años } \\
\text { Tamaño grupo: } 6 \text { individuos } \\
\text { Sexo: } 2 \text { hombres, } 4 \text { mujeres } \\
\text { Residencia: En casco histórico de Córdoba } \\
\text { Concurso: Que sí hayan presentado el patio } \\
\text { al Concurso Municipal de patios, al menos } 3 \\
\text { veces en los últimos } 15 \text { años. }\end{array}$ & $\begin{array}{l}\text { Tipo de vivienda en que viven } \\
\text { - } \quad \text { Vivienda unifamiliar con patio: Mínimo } \\
3 \text { personas. } \\
\text { - } \quad \text { Casa de vecinos con patio colectivo: } \\
\text { Mínimo } 2 \text { ó } 3 \text { personas. }\end{array}$ \\
\hline Gru & $\begin{array}{l}\text { Edad: Más de } 50 \text { años } \\
\text { Tamaño grupo: } 6 \text { individuos } \\
\text { Sexo: } 6 \text { mujeres } \\
\text { Residencia: En casco histórico de Córdoba } \\
\text { Concurso: Que Sí hayan presentado el patio } \\
\text { al Concurso Municipal de patios, al menos } 3 \\
\text { veces en los últimos } 15 \text { años. }\end{array}$ & $\begin{array}{l}\text { Tipo de vivienda en que viven: } \\
\text { - } \quad \text { Vivienda unifamiliar con patio: Mínimo } \\
3 \text { personas. } \\
\text { - } \quad \text { Casa de vecinos con patio colectivo: } \\
\text { Mínimo } 2 \text { ó } 3 \text { personas }\end{array}$ \\
\hline Grupo 5 & $\begin{array}{l}\text { Edad: Entre } 40 \text { y } 55 \text { años } \\
\text { Tamaño grupo: } 6 \text { individuos } \\
\text { Sexo: } 2 \text { hombres, } 4 \text { mujeres } \\
\text { Residencia: En casco histórico de Córdoba } \\
\text { Concurso: Que NO hayan presentado nunca } \\
\text { el patio al Concurso Municipal de patios, o lo } \\
\text { hayan hecho como máximo en } 2 \text { ocasiones en } \\
\text { los últimos } 15 \text { años. }\end{array}$ & $\begin{array}{l}\text { Tipo de vivienda en que viven: } \\
\text { - } \quad \text { Vivienda unifamiliar con patio: } 2 \text { ó } 3 \\
\text { personas. } \\
\text { - } \quad \text { Casa de vecinos con patio colectivo: } 2 \\
\text { ó } 3 \text { personas } \\
\text { - Viviendas unifamiliares modernas ado- } \\
\text { sadas, incorporadas en una sola estruc- } \\
\text { tura inmobiliaria con patio colectivo: al } \\
\text { menos } 2 \text { personas }\end{array}$ \\
\hline
\end{tabular}

Fuente: Elaboración propia.

en contacto con la naturaleza y una forma de imbuirse de la vida agrícola que allí se desarrolla, ya que al cuidar las plantas se ejercen labores similares a las de un agricultor. Los patios son considerados por sus habitantes como reductos de una naturaleza perdida en el tránsito del campo a la ciudad. Esto tiene consecuencias culturales importantes al haberse mantenido la persistencia de prácticas y conocimientos ligados al mundo rural que hoy Ilamaríamos ecológicos o sostenibles. El patio también facilita el contacto con los ciclos climáticos anuales y el conocimiento de sus fenómenos. Se trata de sensaciones que se reconocen en todos los grupos, pero que cada uno expresa con un cierto sentido particular.

$H$ : El patio te permite no salir a la calle. Entonces, cuando Ilegan los cuatro o cinco 
meses de estos de verano horribles aquí, el patio te mantiene la temperatura de la casa fresquita. (...) y estás con el cielo encima y estás con plantas y estás al aire libre (E5).

$M$ : Yo creo que para la gente que tiene un patio, las flores son las niñas de tus ojos. Es como si tienes un perro y cuando llegas a casa lo sacas a pasear, lo lavas, le das de comer, pues las flores igual, las tienes que regar, poner el abono. A mí que me gustan las flores, yo siempre estoy pendiente de que tengan su agua en verano y la luz suficiente en invierno (Grupo 1).

Para el grupo de residentes jóvenes adultos (G.3), tener una casa con patio y vivir en ella es como tener un encuentro continuo con la naturaleza. Es una naturaleza mitificada, construida y dominada por ellos mismos, que la ponen a su servicio y eliminan sus riesgos. El patio y sus plantas representan un vergel particular, un pequeño reino vegetal puesto al servicio del hombre urbano, que a pesar de toda su evolución técnica no puede prescindir de las sensaciones que le reporta el contacto con la naturaleza.

M: Porque nosotros lo que tenemos es un pequeño vergelito en el centro de la ciudad, en una casa.

M: Tener una casa patio, significa tener algo único, que no tiene nada que ver con otro tipo de casa (Grupo 3).

En definitiva, las plantas son el elemento necesario para que un patio pueda considerarse un "auténtico" patio, es decir, constituyen una referencia fundamental en la definición de lo que es un patio cordobés. Para muchos de los entrevistados este criterio de autenticidad pasa por la existencia de vegetación, un elemento indisociable al patio sin la cual este perdería toda su entidad.

La casa con patio y sus plantas, significa para los entrevistados y los grupos de discusión, el aislamiento del entorno urbano, el retorno a la naturaleza y el reencuentro consigo mismo. El patio y su casa aíslan del ruido motorizado del tráfico urbano, favorece el contacto directo con los fenómenos naturales y les permite disfrutar de sus sensaciones sin salir del entorno urbano. Es como vivir en la naturaleza sin salir de esa ciudad.
H: Hombre, esa sensación de, por ejemplo, levantarte por la mañana. Por ejemplo, mi casa no da a la calle, entonces es una casa interior y no sientes ruidos de motos, no sientes ruidos de coches [...].

$H$ : Ver llover en el patio que es superagradable. Salir, desayunar en el porche o en el salón o en el patio.

$H$ : Esas son sensaciones que mucha gente me pregunta: "bueno, ¿qué?". "Mire usted: ¿explíqueme usted la sensación que siente cuando está en su patio?". Y digo: "mire usted, es una sensación muy difícil de explicar...

M: Tienes que vivirlo (Grupo 3).

El grupo de mujeres mayores (G.4) no ha sido muy expresivo respecto a la imagen del patio como reproducción de la naturaleza, pero su satisfacción por el contacto con las plantas sí lo ha sido a lo largo de buena parte del discurso. Su amor y afecto por ese contacto se manifiesta sobre todo a través del conocimiento de cómo afectan los fenómenos climáticos a sus plantas y las técnicas que han desarrollado para evitar que sufran daños por ellas. Por eso, las mujeres mayores son las que proyectan con más intensidad una imagen de cuidadoras, que desarrollan tareas que son una prolongación de los cuidados maternales que ya ejercieron en otra época.

El patio, como contacto con la naturaleza, es para ellas un espacio de libertad que contraponen al encarcelamiento de la vida en los edificios de pisos. Si bien son conscientes de no vivir en la naturaleza, el patio les reproduce y les proporciona sensaciones parecidas a las del ambiente natural, pero sin sus riesgos, obteniendo una seguridad que valoran. Su principal motivo de satisfacción proviene de tener una naturaleza controlada y sin peligros, que les ayuda a vivir alejadas del bullicio de la urbe y les relaja del estrés cotidiano. Es decir, el patio tiene también un significado terapéutico para ellas.

M: El patio de mi casa me da la vida.

M: Yo no sabría vivir sin un patio, ¿eh?

M: Yo tampoco.

M: Yo tampoco.

M: ... Yo, eso de salir por la mañana, abrir mi puerta y salir al patio...

M: Yo también, yo también. 
M: ...eso me da a mí la vida. Yo, encerrada en un piso, yo, es que no puedo, vamos. (Grupo 4).

El grupo de residentes no concursantes (G.5) es el que conforma un discurso significativamente más naturalista, aunque de modo más inconsciente que el resto de los grupos. Para este grupo el patio se define por la presencia de las plantas, por el acuerdo comunitario y por el rechazo a la ornamentación artificial con plantas de plástico. Esto último es una idea específica del grupo que se encuadra dentro de sus deseos de conservar la tradición de las casas con patio y sus barrios, y que explican por qué existen claras diferencias entre las antiguas casas con patio de vecinos y las casas con patio modernas.

Este grupo, junto con el de las mujeres mayores, se sienten dependientes de sus patios y de sus plantas, y manifiesta no poder prescindir de él, porque el patio es una reproducción a pequeña escala de la naturaleza que estimula sus sentidos y con la que experimentan sensaciones que llenan sus vidas.

M: Que yo siempre he vivido en casas, he tenido patio y yo, pues, necesito el patio. Yo me da igual tener una habitación más chica pero necesito el desahogo del patio, de la terraza. Es que lo necesito, aunque la habitación sea más chica, pero a mí, para mí salirme al patio, pues, para mí eso es... (Grupo 5).

Los residentes en casas con patio, tanto concursantes como no concursantes, necesitan rodearse de sensaciones naturales. Por eso extienden la defensa de las casas con patio hacia sus entornos urbanos. No se imaginan sus calles llenas de bloques de pisos, ni las desean, porque significaría la destrucción de ese especial entorno urbano. $Y$ es que al igual que su casa con patio es una isla de naturaleza en un núcleo urbano, su barrio histórico debe continuar siendo una isla en el conjunto de la ciudad. Deben evitar que se mezclen ambos entornos, porque cada tipo de vivienda debe estar ubicada en su espacio específico, sin que vean alguna posibilidad de que se produzca una fusión entre ellos.

Para apoyar al análisis cualitativo de la investigación, y analizar hasta qué punto el contacto de los propietarios con la naturaleza de sus patios es importante, se realizó una pequeña encuesta de dos preguntas a una muestra aleatoria de casas con patio del casco histórico de Córdoba. La primera pregunta analiza las preferencias que tienen los propietarios para pasar su tiempo libre en contacto con la naturaleza (Figura $N^{\circ}$ 6). El 42,6\% de las personas que mantienen o cuidan los patios prefieren pasar su tiempo libre en su propio patio, mientras que el $21,3 \%$ lo prefieren

Figura $\mathrm{N}^{\circ} 6$

Preferencia para pasar el tiempo libre en contacto con la naturaleza.

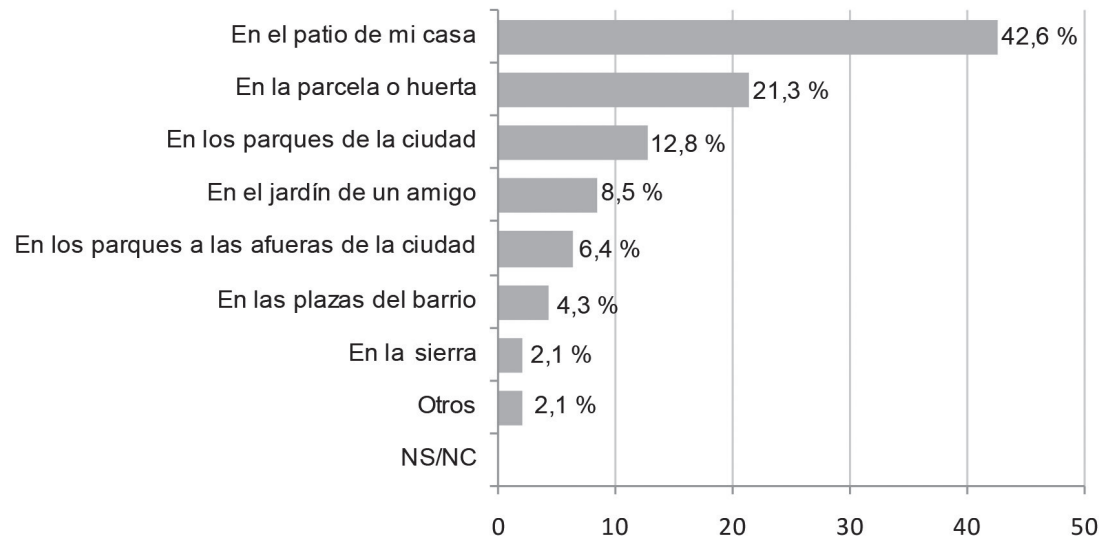

Fuente: Elaboración propia. 
hacer en su parcela o huerta a las afueras de la ciudad. Los parques en la ciudad no suponían una atracción para los ciudadanos que habitan en casas con patio, ya que tan solo el $12,8 \%$ de los encuestados deciden ir a los parques urbanos a pasar su tiempo libre.

Podemos indicar, por tanto, que para los propietarios de las casas con patio de la ciudad de Córdoba, las áreas verdes privadas, incluyendo las parcelas o huertas de recreo, son la primera y segunda elección para pasar sus ratos libres en contacto con la naturaleza.

Estos resultados corroboran los estudios de Breuste et al. (2003), y de Priego et al. (2009), los cuales ponían de manifiesto la importancia que atribuyen los propietarios de determinados espacios verdes privados a su entorno natural más inmediato.

Para muchas personas y disciplinas de las ciencias ambientales, el concepto y definición de naturaleza difiere ampliamente entre si, sin embargo, para los residentes de casas con patio de Córdoba, la naturaleza doméstica es otra forma de entender la naturaleza en todo su concepto y amplitud. Para corroborar la importancia que los residentes de casas con patio le dan a la naturaleza de sus casas, se realizó una segunda pregunta sobre el lugar donde frecuentemente los residentes de este tipo de casas observaban la naturaleza
(Figura $\mathrm{N}^{\circ} 7$ ). De entre los ítems incorporados, el $24,4 \%$ de los entrevistados indicaron que observaban la naturaleza cuando salían a sus patios, y cuando estaban de excursión en algún río, lago o embalse.

Estos resultados ponen en valor la naturaleza privada de las casas como una nueva forma de entender la naturaleza urbana. Para los residentes de las casas con patio, el concepto de naturaleza engloba no solo aquellos espacios verdes prístinos o poco alterados, sino que también una naturaleza domesticada y bajo el control de sus cuidadores.

Una naturaleza que la relacionan con la calidad de vida, ya que se sienten privilegiados en sus hogares por varios motivos. Tener casas con patio es un privilegio frente a una población que ya no tiene capacidad para valorar ese tipo de vida, y en la que el esfuerzo para mantener sus condiciones es una fuente más de satisfacción y placer.

M: Porque a mí, mucha gente, cuando entra a la casa, y ven el patio, me dicen, lo primero que me dicen ¡cuánto trabajo! No te dicen qué bonito, qué espacio, qué flores... No, no, ¡cuánto trabajo! Pues vale, para ti es mucho trabajo, pero para mí merece la pena, es tranquilidad, placer, descanso, un montón de cosas (Grupo 3).

Figura $\mathrm{N}^{0} 7$

Lugar donde observa la naturaleza.

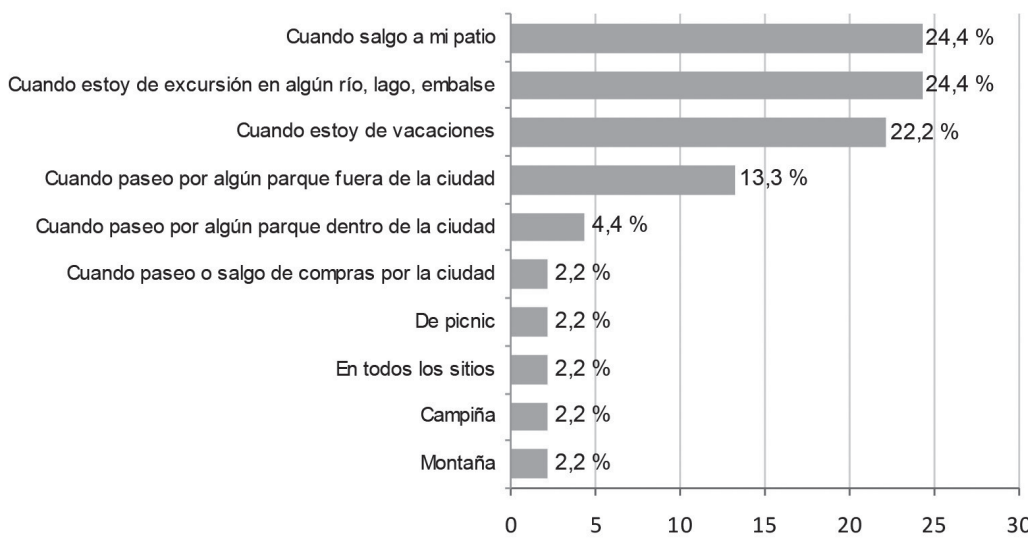

Fuente: Elaboración propia. 
La calidad de vida que se atribuye al hecho de vivir en las casas con patio, es fundamentalmente consecuencia de dos factores: las sensaciones experimentadas por el contacto con la naturaleza del patio y la proyección y reconocimiento social que se obtiene de la participación en el concurso de patios.

Para las mujeres mayores (G.4), el patio es calidad de vida porque les permite sentirse en contacto continuo con la naturaleza y así mejorar su bienestar por el hecho de vivir en un entorno natural con riesgos controlados, que las mantiene alejadas del bullicio de la ciudad y les relaja del estrés cotidiano. Es calidad de vida para los residentes no concursantes porque la naturaleza del patio les posibilita una vida relajada, un ambiente de tranquilidad y un microclima que les produce bienestar en su vida diaria.

M: Yo eso de ver la fuente de mi patio, las flores, y sentarte allí un poquito. Para mí es que es mi rincón de relax. Que es que yo a lo mejor me pongo muy agobiada por lo que sea, me salgo al patio, me fumo un cigarrito, que yo fumo, me siento tranquilita, y oigo... Y cuando entro soy otra persona. Se me va a mí el mal rollo que tengo en la cabeza (Grupo 4).

Para los grupos de entrevistados que presentan el patio a concurso, aumenta su calidad de vida porque obtienen relaciones sociales y reconocimiento social, además de una proyección social de sus actividades cotidianas. A través del concurso de patios se entra en contacto y comunicación con personas que reportan placer cuando expresan su admiración por la labor. Es un reconocimiento social que les hace sentirse felices y realizados y que eleva su calidad de vida porque permite un mejor desarrollo de su personalidad. Además, en el caso concreto de las mujeres mayores, la proyección social del concurso refuerza su rol familiar, porque el cuidado del patio es una tarea análoga a la del cuidado de la propia familia. De ese modo se refuerza este valor, y ello contribuye a sentir una mejor autoestima que se traduce en calidad de vida.

M: ...y luego la otra cosa que nos gustaba era que, por desgracia, los niños ya hoy no pueden jugar en la calle, y este patio permite que los niños no tengan que estar encerrados en casa, y puedan relacionarse con otras personas, y hablamos con las vecinas, con sus madres, y comentamos nuestras cosas.

M: Hombre, la calidad de vida es diferente (Grupo 3).

Para los no residentes en casas con patio (G.1), consideran que los que viven en casas con patio tienen una mejor calidad de vida que los que viven en otro tipo de vivienda sin patio o jardín, y esto lo atribuyen al continuo contacto que tienen con la naturaleza del patio y al cuidado de este.

Para estos jóvenes, el placer que se obtiene cuando se vive en una casa con patio es lo que explica la mejor calidad de vida de estas personas. Y eso deriva de las sensaciones que experimentan cuando visitan los patios durante el concurso. Entonces, la visita les despierta el deseo y la aspiración de lograr una casa con patio.

M: Sentarse en el patio, aunque sea de visita, es maravilloso, eso no tiene precio, es una sensación que nosotros no la vivimos continuamente porque no tenemos casa con patio, pero es que es impresionante, es impagable [...].

$H$ : Es un poco como cuando vas a la playa, que luego lo recuerdas y quieres volver. Pues con el patio igual, que en ese momento desconectas (Grupo 1).

Para los adultos no residentes en casas con patio (G.2), la naturaleza del patio es una fuente de placer, pero también lo es su cuidado, ya que lo ven como una de las mejores sensaciones que provoca la vida en una casa con patio. Por esa percepción, que tiene mucho que ver con las experiencias que vivieron en su infancia (muchos de ellos vivieron anteriormente en casa con patio), las casas con patio y su contexto urbano les estimula el deseo de conseguir un estilo de vida alternativo al que tienen en sus viviendas de pisos, y que se acentúa cuando se alude a los domicilios situados en zonas ruidosas y que son lo opuesto a la tranquilidad que representa una casa con patio.

M: Y la tranquilidad, tú sabes la tranquilidad que es no escuchar nada, porque hay muy 
poco tráfico. Yo es que vivo en una avenida que hay tanto tráfico... A mí me encanta la tranquilidad, yo voy andando a todos lados y el pasear por la parte antigua... Yo callejeo mucho y eso de meterte por las calles y oír mi propio paso y sin oír nada de nada, me encanta (Grupo 2).

En todos los grupos también se han reconocido otros factores positivos de las casas con patio que significan calidad de vida. Uno de ellos es el barrio, que en todos los grupos se reconoce que reproduce en una mayor escala las ventajas de la vida tranquila y relajada de las casas con patio. Es como si las sensaciones vitales que produce el patio se extendieran más allá de sus fronteras y, junto con las otras casas, se creara un barrio acorde con su estilo de vida. Es en el contexto del barrio y la calle en el que se produce la relación con los vecinos, tanto con motivo de los cuidados del patio como porque el barrio en sí promueve la relación vecinal, al quedar el casco histórico apartado del bullicio del tráfico de otras zonas urbanas.

Otro de los aspectos que mejora la calidad de vida es la movilidad física de los moradores, que se ve favorecida por la estructura de las casas con patio. De este modo, la actividad continua que supone cuidar un patio, combate el sedentarismo, reduce el estrés y favorece hábitos de vida saludable, tanto físicos como psíquicos.

Por último, y en relación a la calidad de vida, podemos considerar la importancia que los residentes le dan a las relaciones sociales, entre los vecinos, derivadas del cuidado y ornamentación de los patios. Las técnicas que requieren los cuidados de los patios para conservarlos en buenas condiciones y los esfuerzos realizados para ello son una fuente de intercambio de conocimientos y recursos técnicos y materiales que los participantes en los grupos asocian indiscutiblemente con un motivo de convivencia con los vecinos. En unos casos con los vecinos del patio común y en otros con los vecinos de casas unifamiliares.

\section{Consideraciones finales}

Las conclusiones de esta investigación reflejan la importancia que tiene la cultura social o patrimonial en el uso, percepción y valoración de la naturaleza privada. Resultados como los expuestos en esta investigación vienen a demostrar que el contacto con la "naturaleza privada" de los patios de Córdoba llegan a aportar más beneficios que el contacto con otro tipo de "naturaleza urbana pública" (parques, jardines, etcétera), ya que son el primer lugar que prefieren los usuarios de las casas con patio, para estar en contacto con la naturaleza.

Además de los numerosos beneficios que de por sí la naturaleza aporta al ciudadano, el contacto privado de la naturaleza en los patios de Córdoba contribuye de una forma importante al aumento de las relaciones sociales de sus propietarios, tanto a nivel familiar como vecinal, a la autoestima de sus propietarios, al enraizamiento en la comunidad y a la identidad sociocultural, entre otros. En las casas con patios encontramos varios ámbitos de relaciones y actividades que les dan sentido y significado propio. En el ámbito privado, las posibilidades que ofrece la casa con patio son numerosas. Entre ellas, cabría destacar el significado que tiene el patio como lugar de contacto con la naturaleza que estimula los sentidos: por su contemplación, por el contacto con las plantas al ser cuidadas, por el acercamiento y protección de los fenómenos meteorológicos, así como por ser un espacio para el relax y el descanso. En definitiva son actividades que se viven como privilegio personal y que se revalorizan cuando se comparan con el estilo de vida en otro tipo de viviendas, como los bloques de pisos. Un privilegio que es percibido como calidad de vida y que llega a tener hasta propiedades terapéuticas.

Por otro lado, y además de los beneficios psicosociales que los patios de Córdoba aportan a sus dueños, las casas con patio son un lugar en el que armonizar una vida comunal y social, un lugar apropiado en los que satisfacer el deseo de estar en contacto con la naturaleza sin tener que salir fuera de la casa. En los barrios históricos, donde predominan las casas con patios, los parques urbanos no son el referente natural más cercano que tiene la población para tener acceso a la naturaleza. De este modo, este tipo de viviendas rompen la dinámica social predominante en el contexto urbano, que es la de desplazarse 
para estar y beneficiarse del contacto directo con la naturaleza.

Finalmente, consideramos que aunque son necesarias nuevas investigaciones en el estudio de la naturaleza urbana en zonas mediterráneas, y con un fuerte carácter histórico, esta investigación pone en tela de juicio a los indicadores de ámbito internacional en materia de espacios verdes urbanos. Numerosas investigaciones e instituciones, como la Organización Mundial de la Salud, consideran que las ciudades deben de tener un número mínimo de metros cuadrados, per cápita, de espacios verdes públicos para que las personas puedan desarrollarse plenamente. Sin embargo, estas investigaciones no suelen incluir en este indicador la presencia y contacto que los ciudadanos hacen de sus espacios verdes privados. Con esta investigación queda demostrado que en muchas ciudades mediterráneas la presencia de espacios verdes privados supone una alternativa a los espacios públicos, y que, en muchos casos, aportan más satisfacción que estos últimos. Por lo tanto, esta investigación plantea la necesidad de revisar estos indicadores internacionales, para que se permitiera comparar, de forma más equitativa, países que por su situación geográfica y condiciones climatológicas disponen de grandes extensiones de espacios verdes urbanos, con otros países, como los mediterráneos, cuyas condiciones climatológicas han propiciado que, a lo largo de los siglos, los ciudadanos desarrollen nuevas formas de contacto con la naturaleza.

\section{Referencias bibliográficas}

AYUNTAMIENTO DE CÓRDOBA. La rehabilitación y cualificación del centro histórico en la nueva estrategia urbanística general. Córdoba: Gerencia de Urbanismo, Ayuntamiento de Córdoba, 2009. Disponible en internet: http://www.gmu.ayuncordoba.es/ capitulo-v.-el-casco-historico.html

BEER, A.R. Urban greenspace and sustainability. In: VAN DER VECHT, H. (editor). Sustainable urban development: research and experiments. Delft: University Press, 1994, p. 69-88.

BERMAN, L. How does our garden grow? A guide to community garden success. Toronto: FoodShare Metro Toronto, 1997.
BENZEVAL, M.; JUDGE, K. \& WHITCHCAD, M. Tackling Inequalities in Health. London: Kings Fund, 1995.

BREUSTE, J.; ROJAS, J.; KASPERIDUS, H.D. y PRIEGO, C. Utilización y Manejo de la Naturaleza y del Paisaje en Aglomeraciones Urbanas. Leipzig: UFZ-Bericht, 2003.

CAMILLONI, I.A. \& BARROS, V.R. On the urban heat island effect dependence on temperature trends. Climatic Change, 1997, Vol. 37 , p. 665-681.

CAMPBELL, A.; CONVERSE, P. \& ROGERS, W. The Quality of American Life: Perceptions, Evaluations and Satisfactions. New York: Russell Sage Foundation, 1976.

CHIESURA, A. The role of urban parks for the sustainable city. Landscape and Urban Planning, 2004, Vol. 68, p. 129-138.

COOK, D.I. Trees, solid barriers, and combinations: Alternatives for noise control. In: HOPKINS, G. (editor). Proceedings of the National Urban Forestry Conference. Washington: ESF Publications, 1978, p. 330-339.

COOK, D.I. \& HAVERBEKE, D.F. Trees and shrubs for noise abatement. Research Bulletin, 1971, Vol. 246, p. 1-77.

CROW, T.; BROWN, T. \& DEYOUNG, R. The Riverside and Berwyn experience: contrasts in landscape structure, perceptions of the urban landscape, and their effects on people. Landscape and Urban Planning, 2006, Vol. 75, p. 282-299.

ELLIS, C.D; LEE, S.W. \& KWEON, B. Retail land use, neighbourhood satisfaction and the urban forest: an investigation into the moderating and mediating effects of trees and shrubs. Landscape and Urban Planning, 2006, Vol. 74, p. 70-78.

GOBSTER, P.H. Perception and use of a metropolitan greenway system for recreation. Landscape and Urban Planning, 1995, Vol. 33, p. 401-413.

HILLS, J. Inquiry into Income and Wealth. York: Joseph Rowntree Foundation, 1995. 
HOUGH, M. City Form and Natural Process. London: Routledge, 1989.

JIM, C.Y. \& CHEN, W.Y. Recreation-amenity use and contingent valuation of urban greenspaces in Guangzhou. China. Landscape and Urban Planning, 2006, Vol. 75, p. 81-96.

KAPLAN, R. \& AUSTIN, M.E. Out in the country: sprawl and the quest for nature nearby. Landscape and Urban Planning, 2004, Vol. 69, p.235-243.

KUO, F.E.; SULLIVAN, W.C.; COLEY, R.L. \& BRUNSON, L. Fertile Ground for community: Inner-City Neighborhood Common Spaces. American Journal of Community Psychology, 1998, Vol. 26, № 6, p. 823-851.

LEWIS, C.A. Effects of Plants and Gardening in Creating Interpersonal and Community Well-Being. In: RELF, D. (editor). The Role of Horticulture in Human Well-Being and Social Development: A National Symposium. Portland: Timber Press, 1992, p. 55-65.

LEWIS, C.A. Green Nature, Human Nature. The Meaning of Plants in Our Lives. Chicago: University of Illinois Press, 1996.

MATSUOKA, R.H. \& KAPLAN, R. People needs in the urban landscape: Analysis of Landscape and Urban Planning contributions. Landscape and Urban Planning, 2008, Vol. 84, p. 7-19

MILES, I.; SULLIVAN, W.C. \& KUO, F.E. Ecological restoration volunteers: the benefits of participation. Urban Ecosystems, 1998, Vol. 2. p. 27- 41 .
MILLER, R.H. Urban Forestry: Planning and Managing Urban Greenspaces. Ney Jersey: Prentice Hall, 1988.

ÖZGÜNER, H. \& KENDLE, A. Public attitudes towards naturalistic versus designed landscapes in the city of Sheffield (UK). Landscape and Urban Planning, 2006, Vol. 74, p. 139-157.

PACIONE, M. Urban environmental quality and human wellbeing-a social geographical perspective. Landscape and Urban Planning, 2003, Vol. 65 p. 19-30.

PECK, S.W. \& CALLAGHAN, C. Greenbacks from Green Roofs: Forging a New Industry in Canada. Ottawa: Canadian Mortgage and Housing Corporation, 1999.

PRIEGO, C.; BREUSTE, J. y ROJAS, J. Opinión pública, valoración y uso de espacios naturales en zonas urbanas. Análisis comparado de cuatro experiencias en la ciudad alemana de Halle y las chilenas de San Pedro de la Paz y Talcahuano. Revista Internacional de Sociología, 2009, Vol. 1, p. 199-224.

SMITH, W.H. Urban Vegetation and Air Quality. Syracuse: SUNY, 1978.

SMITH, W.H. Air pollution and Forest. New York: Springer- Verlag, 1990. 